1841 A FOUR-YEAR RETROSPECTIVE STUDY OF THE SAFETY AND EFFICACY OF PALIVIZUMAB IN HIGH RISK INFANTS

doi:10.1136/archdischild-2012-302724.1841

R Abusamra, M Schofield, C Minasian. Department of Paediatrics \& Adolescent Medicine, University College London Hospitals, London, UK

Aim A four year retrospective review of safety and efficacy of Palivizumab in the prophylaxis of respiratory syncytial virus (RSV) in high risk infants.

Background RSV is the leading viral pathogen responsible for hospitalization with lower respiratory tract infection in high risk infants A previous study reported a $55 \%$ reduction in RSV related hospitalizations with passive immunoprophylaxis with Paluvizimab ${ }^{1}$.

Methods A retrospective study of all high risk infants requiring Palivizumab between 2006 and 2010. Inclusion criteria included children under 2 years with chronic lung disease on home oxygen, those with congenital immunodeficiency and infants under 6 months with confirmed congenital heart disease. Children received intramuscular injections of Palivizimab every 28days during the RSV season (October-March). Data was collected on demographics, underlying diagnosis, side-effects and confirmed RSV infection. A cost analysis was also performed

Results Records on 61 patients were examined. 15 children did not meet the study criteria. Data on RSV infection was available for 52/61 patients. Of these children, none of those who received a complete course of prophylaxis developed RSV infection. Reported side-effects were mild with the exception of one child with a seizure disorder who had an increase in fit frequency. Having dedicated clinics for administering Palivizimab, allowed sharing of opened vials between patients and more effective utilization of clinic staff and resources.

Conclusions RSV prophylaxis is safe and effective in preventing RSV infection in high risk infants. Further strategies have been used to reduce drug costs and improve cost-effectiveness.

\section{EFFECT OF NEURONAL EXPRESSION OF HENT1 AND CAFFEINE ON CEREBRAL BLOOD FLOW AND CORTICAL STROKE IN MICE}

doi:10.1136/archdischild-2012-302724.1842

1,2 $\mathrm{H}$ Soylu, 'D Zhang, ${ }^{3} \mathrm{R}$ Buist, ${ }^{1,3,4} \mathrm{M}$ Martin, ${ }^{1} \mathrm{BC}$ Albensi, ${ }^{1} \mathrm{FE}$ Parkinson. ' ${ }^{2}$ harmacology 8 Therapeutics; ${ }^{2}$ Neonatology/Pediatrics; ${ }^{3}$ Radiology, University of Manitoba; ${ }^{4}$ Physics, University of Winnipeg, Winnipeg, MB, Canada

Background Adenosine has neuromodulatory effects in animal stroke models. We previously showed that human equilibrative nucleoside transporter 1 (hENT1) over expression decreases extracellular adenosine action. Caffeine is adenosine receptor antagonist.

Objective To compare the effect of ENT1 over expression on cerebral blood flow (CBF) and subsequent ischemic damage in hENT1 transgenic $(\mathrm{Tg})$ and wild type $(\mathrm{Wt})$ mice following intra-cortical injection of endothelin-1 (ET-1) and giving intraperitoneal (IP) caffeine prior to the ischemic stroke event.

Design/methods 8-10 week old CD1 and Tg mice were stratified as Group A $(\mathrm{n}=25)$, received unilateral single cortical injection of ET-1 and Group B $(n=20)$ received $25 \mathrm{mg} / \mathrm{kg}$ IP caffeine prior to ET-1 injection. CBF was measured at 4 hours and 48 hours and stroke size was measured at 48 hours by MRI.

Results At 4 hours ipsilateral CBF decreased significantly $(p<0.01)$, which was more prominent in $\mathrm{Tg}$ mice and was still evident at 48 hours. ET-1 produced greater infarct size in $\operatorname{Tg}\left(9 \pm 1.1 \mathrm{~mm}^{3}\right)$ than Wt $\left(5.4 \pm 0.8 \mathrm{~mm}^{3}\right)$ mice without given caffeine. However, there was no difference in infarct size between $\operatorname{Tg}\left(6.2 \pm 1 \mathrm{~mm}^{3}\right)$ and $\mathrm{Wt}(6.7 \pm 1$ $\mathrm{mm}^{3}$ ) mice in caffeine injected group.

Conclusions This study showed that hENT1 over expression is associated with increased cerebral infarct size. This genotype difference was not observed in mice received caffeine. These data are consistent with our previous findings that hENT1 Tg mice have reduced basal adenosine levels and reduced ischemia evoked increases in adenosine as compared to Wt mice.

\section{HIGH FREQUENCY OSCILLATION AND PRONE POSITION IN THE EXPERIMENTAL MODEL OF ARDS - CHANGES OF PRESSURE HEMODYNAMIC PARAMETERS}

doi:10.1136/archdischild-2012-302724.1843

J Žurek, P Košut, P Dominik, M Klimovič, M Šeda, M Fedora. Department of Anesthesia and Intensive Care, School of Medicine, Masaryk University, Brno, Czech Republic

Background and Aims High frequency oscillation (HFO) and prone position ventilation have their places in the treatment of an inhomogeneous pulmonary pathology of ARDS. Possible benefits of early used HFO include improved ventilation and oxygenation, reduced risk of ventilator induced lung injury development, lower plasmatic levels of pro-inflammatory cytokines, and therefore reduced mortality of ARDS patients. Prone position ventilation improves oxygenation, but without an impact on mortality or length of hospitalization. Aim of this study was to compare the pressure hemodynamic parameters in experimental models of ARDS ventilated in prone position or by HFO.

Methods Total of 16 experimental piglets were randomised into 2 groups of 8 piglets: PP group ventilated conventionally in prone (18 hours) and supine ( 6 hours) position, HFO group ventilated 24 hours in supine position with HFO. Mean arterial pressure (MAP), central venous pressure (CVP), mean pulmonary artery pressure (mPAP) and pulmonary capillary wedged pressure (PCWP) were measured invasively, indexed systemic (SVRI) and pulmonary (PVRI) vascular resistances were calculated.

Results MAP is lower in HFO group than in PP ( 3 hours: $p=0,001$; 6 hours: $p=0,016$; 9 hours: 0,$007 ; 12$ hours: 0,041$)$. mPAP is higher in PP group ( 3 hours: $p=0,001)$, as well as PVRI $(1$ hour: $p=0,003)$ and SVRI ( 9 hours: 0,027$)$. CVP and PCWP values show no differences between groups.

Conclusions Early used HFO lowers PVRI in first hours of ARDS, PP has milder impact on systemic pressures - combination of both procedures can be beneficial.

Grant Acknowledgment IGA MZČR NS 11100-4

\section{OUTCOME OF EXTREME PREMATURITY: A REVIEW OF RECENT DATA}

doi:10.1136/archdischild-2012-302724.1844

C Bellieni, G Buonocore. Pediatrics and Obstetrics, University of Siena, Siena, Italy

Aim To review the most recent data on the outcome of very premature babies (22-25 weeks gestational age - GA).

Material and Methods We performed a PubMed search using as key words the following "extremely preterm baby" and "outcome". We did not utilise the studies where outcomes were not given in relationship with the exact gestational age at birth. We utilised the studies that reported either survival or health as the examined outcomes.

Results We retrieved 33 papers, 6 of which fulfilled the search criteria. Survival for babies born at 22, 23, 24 and 25 weeks range 27-64, 7-64, 25-79 and 46-89 respectively. Other outcomes are more detailed: the outcomes improve with the GA, and varies according with the type of disability we analyzed. Discussion: several studies are based on few cases, and therefore they have limited value, but some have a huge population and the data they give are very useful and new.

Conclusion With respect to previous data based on populations of babies born in the last century, the present data show an improvement in both survival and neurologic outcome, though both remain severe Current guidelines on resuscitation should be reviewed accordingly. 